\title{
PERSPECTIVES Compassion in digital healthcare
}

\author{
Author: Eddie Kinsella ${ }^{\mathrm{A}}$
}

KEYWORDS: Compassion, empathy, digital health

DOI: 10.7861/fhj.dig-2020-comp

The devastating impact of COVID-19 has changed our day-today reality in a remarkably short timescale. As we move into the NHS recovery and reset mode, the Royal College of Physicians' (RCP's) Patient and Carer Network (PCN) has started the process of considering the detailed implications for patient-centred care within a digital healthcare system. Our focus will be to ensure that the emphasis is upon benefits for patients and carers, and that appropriate safeguards are built in to avoid pitfalls, especially for those who are vulnerable or disadvantaged.

Digital healthcare represents a cultural change as much as a technical change. Many questions arise around privacy, patient safety, those patients whose first language is not English, those people who are not digitally enabled, those with long-term conditions, mental health problems etc. The list is significant and requires careful consideration in terms of communication, training and education.

The central theme is that of ensuring 'the human touch' between clinicians, patients and carers is not lost along the way. Compassionate care is fundamental to any healthcare system. The tragedies arising from COVID-19 serve to remind us starkly that humanity must remain the driving principle.

The central point of the patient-centred model of doctor-patient relationship is that medical competency should not be reduced to technical expertise, but must include relational moral competency, particularly empathy, compassion and trust. ${ }^{1}$

[Compassion includes] empathy, respect, a recognition of the uniqueness of another individual, and the willingness to enter into a relationship in which not only the knowledge but the intuitions, strengths, and emotions of both the patient and the physician can be fully engaged. ${ }^{2}$

Note the emphasis on the softer but crucially important elements of the interaction, and the implication that it's not a 'one-way street'. We must not lose that.

Author: A chair of the RCP Patient and Carer Network, Royal College of Physicians, London, UK
Compassion - both giving and receiving it - entails an emotional response. $^{3}$

It is that emotional commitment (which has been exemplified with the sacrifices made by so many healthcare staff during the pandemic) that we must ensure is not inadvertently diluted by the use of technology. The PCN welcomes the move towards digital healthcare on the basis of a shared decision making and co-production model. The pilot projects associated with the RCP's Future Hospital Programme have demonstrated many good examples of best practice.

Digital solutions that automatically capture and analyse data can provide helpful insights for diagnosis, reporting quality measures, and release more time for those patients who most need it. Digital devices can allow patients to provide continuous feedback, share needs and concerns, and help them to manage their medication regimen and postoperative protocol more effectively.

Most importantly, digital health systems can empower and engage patients, making them co-designers of care and an integral part of the care team. Healthcare professionals become collaborators in a patient's journey to health, while still providing empathy and a human touch in support of patients' wellbeing. The PCN will work closely with the RCP to help strike the right balance between technological efficiency and compassionate care.

\section{References}

1 Kerasidou A. Empathy, compassion and trust balancing artificial intelligence in health care. Bull World Health Organ 2020 [Epub ahead of print].

2 Lowenstein J. The midnight meal and other essays about doctors, patients, and medicine. New Haven: Yale University Press, 1997.

3 Frank AW. The renewal of generosity: illness, medicine and how to live. Chicago: The University of Chicago Press, 2004.

Address for correspondence: Eddie Kinsella, Royal College of Physicians, 11 St Andrews Place, Regent's Park, London NW1 4LE, UK.

Email: eddie.kinsella@btinternet.com 